SLAC-PUB-11897

hep-ph/0606129

June 2006

\title{
Dynamical GUT breaking and $\mu$-term driven supersymmetry breaking
}

\author{
Ryuichiro Kitano \\ Stanford Linear Accelerator Center, Stanford University, Stanford, CA 94309
}

\begin{abstract}
Models for dynamical breaking of supersymmetric grand unified theories are presented. The doublet-triplet splitting problem is absent since the Higgs doublet superfields can be identified with the massless mesons of the strong gauge group whereas there are no massless states corresponding to the colored Higgs fields. Various strong gauge groups $\mathrm{SU}\left(N_{c}\right), \operatorname{Sp}\left(N_{c}\right)$ and $\mathrm{SO}\left(N_{c}\right)$ are examined. In a model with $\mathrm{SO}(9)$ strong gauge group, adding the $\mu$-term for the Higgs fields triggers to break supersymmetry in a meta-stable vacuum. The pattern of the supersymmetry breaking parameters is predicted to be the gauge-mediation type with modifications in the Higgs sector.
\end{abstract}




\section{Introduction}

The Higgs boson, which has not been observed yet, is the most mysterious particle in the standard model although it plays the most important role: electroweak symmetry breaking and the origin of the fermion masses. Successful electroweak symmetry breaking needs the negative mass squared for this particle and the size must be anomalously small compared to the cut-off scale of the theory. This situation motivated us to consider the supersymmetric standard model to protect the mass parameter from the large quantum correction, but the Higgs particle is still left mysterious. The Higgs boson (or Higgsino) mass parameter, the $\mu$-term, cannot be protected by gauge symmetry or supersymmetry (SUSY) although the quantum correction is successfully removed.

Embedding the supersymmetric standard model into grand unified theories (GUT), motivated by the gauge coupling unification [1], makes the Higgs particle more mysterious. Even for the smallest group for grand unification, SU(5) [2], the Higgs fields do not fit into a complete multiplet of the symmetry group, and therefore we need extra particles to appear at the GUT scale $M_{G} \sim 10^{16} \mathrm{GeV}$. The mysterious feature is that, first, it is not easy to realize the situation with nearly massless Higgs fields in models with the GUT symmetry breaking by the Higgs mechanism. The splitting of the masses between the Higgs fields and extra particles must be done by the coupling through the fields whose vacuum expectation values (VEV) break the GUT symmetry group, but in simple models it gives masses for the Higgs fields of the order of $M_{G}$. This is the famous doublet-triplet splitting problem. Another mysterious feature of SUSY-GUT models is the absence or suppression of the dimension-five proton-decay operators. For example, in the simplest SU(5) model, the Higgs particles are embedded into the $\mathbf{5}$ and $\overline{\mathbf{5}}$ representations which contain two colored partner of the Higgs fields $H_{C}$ and $\bar{H}_{C}$. If those colored Higgs fields have masses by pairing each other, i.e., $W \ni m_{C} H_{C} \bar{H}_{C}$, the dimension-five operators suppressed by the scale $m_{C}$ are generated by integrating out $H_{C}$ and $\bar{H}_{C}$ [3]. It has been studied that the colored Higgs mass $m_{C}$ has to be quite large $m_{C} \gtrsim 10^{17} \mathrm{GeV}$, which is disfavored by the unification of the gauge couplings [4.

The above two problems, the doublet-triplet splitting and the proton decay, are actually related and there is a simple solution to those problems. If we are to avoid the dimension-five proton-decay operators such as $Q Q Q L$ in the superpotential, with $Q$ and $L$ being the quark and lepton doublets, an easy way is to impose symmetry where matter fields are charged, e.g., both $Q$ and $L$ have charge unity. In SU(5) SUSY GUTs, this means that both of the Higgs fields in $\mathbf{5}$ and $\overline{\mathbf{5}}$ representations have charge -2 in order to have Yukawa interactions, and thus the mass term is forbidden. Now, to give mass terms for colored Higgs fields while keeping symmetry to 
be unbroken we need to introduce another pair of $\mathbf{5}$ and $\overline{\mathbf{5}}$ field which have charge +2 , but in this case, we have either zero or four Higgs-doublet fields in low energy which is unacceptable. Of course, adding further pair of $\mathbf{5}$ and $\overline{\mathbf{5}}$ field with charge -2 results in extra massless colored Higgs fields. Therefore, in order to have only two Higgs doublets while forbidding the proton decay by continuous symmetry, we need to repeat the procedure of adding $\mathbf{5}$ and $\overline{\mathbf{5}}$ forever and it ends up with infinite number of Higgs fields in $\mathbf{5}$ and $\overline{\mathbf{5}}$ representations [5].

While the infinite number of particles sounds unreasonable in field theory, it is quite possible to realize this situation in models with extra-dimensions. The infinite number of particles are identified with the Kaluza-Klein tower of the fields which are propagating into the bulk of the extra-dimension. Indeed, simple GUT models have been constructed in higher dimensional space-time, where the boundary condition breaks the GUT symmetry and there is no doublettriplet splitting [6] or the proton decay problem [7]. In this picture, the Higgs particles become less mysterious. They are just a bulk field.

On the other hand, there is another familiar mechanism of having infinite tower of particles in field theory, that is actually happening in QCD. When asymptotically free gauge theory becomes strong in low energy, the effective theory below that scale is described by gauge-singlet particles such as mesons and baryons. These particles have also infinite tower of excitation states. This fact naturally leads us to think of the possibility of realizing Higgs particles as composite fields in some strongly coupled gauge theory which breaks GUT symmetry dynamically. This question is also interesting from the viewpoint of the AdS/CFT correspondence [8, 9]. The extradimensional GUT models above may be interpreted as a dual picture of the strongly coupled theory. The explicit gauge symmetry breaking in the extra-dimensional picture may be justified by the presence of viable dynamical GUT breaking models.

Constructing GUT models associated with a strongly coupled gauge theory have been attempted by the group of Hotta, Izawa and Yanagida [10, 11, 12, 13]. Various gauge groups for the strong interaction, $\mathrm{SU}(3)(\times \mathrm{U}(1))$ [10, 11], $\mathrm{SU}(5)$ [13] and $\mathrm{SO}(6)$ 12], were considered. It was found that the doublet-triplet splitting can be easily realized via the missing partner mechanism while preserving (anomalous) U(1) symmetry which forbids the dimension-five proton decays. Along the similar line, a model with $\operatorname{Sp}(2)$ gauge group has also been constructed recently in Ref. [14 where the model is quite simplified. (Our convention is such that $\operatorname{Sp}(1) \simeq \mathrm{SU}(2)$.) The model consists of six flavor quarks of $\operatorname{Sp}(2)$ and five of flavors are identified with $\mathbf{5}$ and $\overline{\mathbf{5}}$ representation fields of the SU(5) GUT. The other flavor turns out to be (constituent of) the Higgs doublets in low energy. In the model, the conformal field theory (CFT) nature of the $\mathrm{Sp}(2)$ interaction above the GUT scale plays a crucial role. A similar approach in the warped extra-dimension can also be found in Ref. [15]. 
In this paper, we consider the generalization of the $\operatorname{Sp}(2)$ model. We find that the models with $\operatorname{Sp}\left(N_{c}\right)$ with $N_{c}=2$, and $\mathrm{SO}\left(N_{c}\right)$ with $6 \leq N_{c} \leq 9$ work for dynamical GUT breaking with having massless doublet Higgs fields, and no viable $\mathrm{SU}\left(N_{c}\right)$ group is found under assumptions on the particle content and superpotential. Of particular interest is the case with $\mathrm{SO}\left(N_{c}\right)$ gauge group. There is no exotic particle left massless without adding superpotential terms to remove those particles.

With the success of the doublet-triplet splitting while forbidding the proton decay, the final missing piece for the Higgs mystery is the finite $\mu$-term. In the gravity mediated supersymmetry breaking scenario [16], it is possible to obtain a correct size of the $\mu$-term in a simple way [17]. However, we find an alternative interesting possibility in the $\mathrm{SO}(9)$ model. Instead of solving the $\mu$-problem, if we add a small $\mu$-term in the superpotential, supersymmetry breaks down at the intermediate scale $F \sim \mu M_{G}$. Although this is not the true vacuum, it is shown to be meta-stable [18]. It is amusing that the $\mu$-term can drive the supersymmetry breaking, which is the opposite direction to the usual thought. The smallness of the $\mu$-term is "explained" by demanding low supersymmetry breaking scale. The Higgs fields can be responsible not only for electroweak symmetry breaking but also for GUT and supersymmetry breaking.

We start the discussion of the dynamical GUT breaking in Section 2, there the general setup is defined. We analyze a successful model, the $\mathrm{SO}(9)$ model, in Section 3. In section 4, the mechanism of $\mu$-term driven supersymmetry breaking is presented and we discuss mediation of the supersymmetry breaking to our sector.

\section{Dynamical GUT breaking}

In Ref. [14], a simple model for the dynamical GUT breaking is constructed based on $\operatorname{Sp}(2)$ gauge theory. We study generalization of the model with various gauge groups: $\mathrm{SU}\left(N_{c}\right), \operatorname{Sp}\left(N_{c}\right)$ and $\mathrm{SO}\left(N_{c}\right)$. Models with $\mathrm{Sp}\left(N_{c}\right)$ and $\mathrm{SO}\left(N_{c}\right)$ with certain range of $N_{c}$ is found to be viable but no viable $\mathrm{SU}\left(N_{c}\right)$ group is found. Although they are not successful models, we start with the discussion of the $\mathrm{SU}\left(N_{c}\right)$ models in which we can see the essential features of this class of models.

\section{$2.1 \mathrm{SU}\left(N_{c}\right)$ models}

The model consists of six flavors and five of them carry SU(5) GUT quantum numbers as listed in Table 1. The quarks and leptons in the standard model are not charged under $\mathrm{SU}\left(N_{c}\right)$ and 


\begin{tabular}{ccc} 
& $\mathrm{SU}\left(N_{c}\right)$ & $\mathrm{SU}(5)_{\mathrm{GUT}}$ \\
\hline \hline$Q$ & $N_{c}$ & $\mathbf{5}$ \\
$\bar{Q}$ & $\bar{N}_{c}$ & $\overline{\mathbf{5}}$ \\
$T$ & $N_{c}$ & $\mathbf{1}$ \\
$\bar{T}$ & $\bar{N}_{c}$ & $\mathbf{1}$ \\
\hline \hline
\end{tabular}

Table 1: The particle content of the $\mathrm{SU}\left(N_{c}\right)$ model.

unified usually as $\mathbf{1 0}$ and $\overline{\mathbf{5}}$ of $\mathrm{SU}(5)_{\text {GUT }}$. We introduce superpotential for $Q$ and $\bar{Q}$ :

$$
W=m \operatorname{Tr}(Q \bar{Q})-\frac{1}{M} \operatorname{Tr}[(Q \bar{Q})(Q \bar{Q})]+\cdots,
$$

where $(Q \bar{Q})$ is the $\mathrm{SU}\left(N_{c}\right)$ singlet $5 \times 5$ matrix, and ' $\ldots$ ' represents other higher dimensional operators such as $(\operatorname{Tr}(Q \bar{Q}))^{2}$ and those are not important for the discussion. It is essential for the masslessness of the Higgs fields that the superfields $T$ and $\bar{T}$ do not have superpotential at tree level since the Higgs fields will be identified with the meson fields $H \sim Q \bar{T}$ and $\bar{H} \sim \bar{Q} T$.

Before the analysis at the quantum level, it is helpful for the understanding of the model to discuss what happens at the classical level. The classical analysis is valid for $\Lambda \ll M_{G}$ with $\Lambda$ being the dynamical scale of $\mathrm{SU}\left(N_{c}\right)$. In this case, the picture becomes models with product group unification [19]. At the classical level, there are vacua with $\operatorname{rank}(Q \bar{Q})=0$ to $\min \left[5, N_{c}\right]$ with satisfying the conditions of $F_{Q}=F_{\bar{Q}}=0$. We are interested in the vacuum with $\operatorname{rank}(Q \bar{Q})=2$ :

$$
(Q \bar{Q})=\left(\begin{array}{ccccc}
0 & & & & \\
& 0 & & & \\
& & 0 & & \\
& & & v^{2} & \\
& & & & v^{2}
\end{array}\right)
$$

where $v^{2}=m M / 2$. At the vacuum, the $\mathrm{SU}\left(N_{c}\right) \times \mathrm{SU}(5)_{\text {GUT }}$ gauge symmetry is broken down to $\mathrm{SU}\left(N_{c}-2\right) \times \mathrm{SU}(3)_{C} \times \mathrm{SU}(2)_{L} \times \mathrm{U}(1)_{Y}$ for $N_{c} \geq 3$ and the electroweak $\mathrm{SU}(2)_{L} \times \mathrm{U}(1)_{Y}$ is the diagonal subgroup of those in $\mathrm{SU}\left(N_{c}\right)$ and $\mathrm{SU}(5)_{\text {GUT }}$. Note that the vanishing components are not a consequence of the fine-tuning. The corresponding components in $Q$ and $\bar{Q}$ are charged under unbroken gauge symmetry and that ensures the absence of the linear terms in the potential, i.e., stable (or flat directions). Since the low energy $\mathrm{SU}(2)_{L} \times \mathrm{U}(1)_{Y}$ partly comes from $\mathrm{SU}\left(N_{c}\right)$, two of the components in $T$ and $\bar{T}$ transforms exactly the same way as the Higgs fields in low energy whereas there is no colored component in $T$ or $\bar{T}$. Therefore the doubletriplet splitting problem and proton decay mediated by the colored Higgs are absent. The rest of components in $T$ and $\bar{T}$ are fundamental and anti-fundamental representations of $\mathrm{SU}\left(N_{c}-2\right)$ and charged under $\mathrm{U}(1)_{Y}$. All the components in $Q$ and $\bar{Q}$ are either eaten by gauge fields of 
broken symmetry or obtain masses from the superpotential. The fate of the exotic particles in $T$ and $\bar{T}$ depends on the dynamics of the unbroken $\mathrm{SU}\left(N_{c}-2\right)$ group below the dynamical scale. Of course, with $\Lambda \ll v$, this is not a "unified" model. The three gauge coupling constants do not meet at the GUT scale since the embeddings of $\mathrm{SU}(3)_{C}$ and $\mathrm{SU}(2)_{L} \times \mathrm{U}(1)_{Y}$ are different. The real unification picture arises when $\Lambda \gtrsim v$, where the quantum effect is important.

At the quantum level, the low energy physics is not very different, but some of the vacua are lifted. In particular, it is interesting to note that the dynamical symmetry breaking has to happen once we take into account the quantum effect [12]. With the above superpotential, the low energy theory of the $\operatorname{rank}(Q \bar{Q})=0$ vacuum is $\mathrm{SU}\left(N_{c}\right)$ with one flavor $T$ and $\bar{T}$, which does not have a ground state [20]. The stability of the classical vacua of $\operatorname{rank}(Q \bar{Q})=2$ depends on $N_{c}$. We show below that there is no $N_{c}$ which is viable for low energy phenomenology.

For $N_{c}=2$, theory is not asymptotically free and classical analysis is valid in low energy. However, in this case, the vacuum in Eq. (2) breaks the gauge symmetry into $\mathrm{SU}(3) \times \mathrm{SU}(2)$ which is not acceptable.

For $N_{c}=3$, the quantum effect is easier to analyze in the dual gauge theory [21]. It is again $\mathrm{SU}(3)$ gauge theory but with superpotential:

$$
\begin{aligned}
W & =m \operatorname{Tr} M_{Q \bar{Q}}-\frac{1}{M} \operatorname{Tr}\left(M_{Q \bar{Q}} M_{Q \bar{Q}}\right)+\cdots \\
& +\frac{1}{\hat{\Lambda}} \bar{q} M_{Q \bar{Q}} q+\frac{1}{\hat{\Lambda}} H \bar{q} t+\frac{1}{\hat{\Lambda}} \bar{H} q \bar{t}+\frac{1}{\hat{\Lambda}} S t \bar{t}
\end{aligned}
$$

where mesons are identified with the quark bilinears in the original (electric) theory: $M_{Q \bar{Q}} \sim Q \bar{Q}$, $H \sim Q \bar{T}, \bar{H} \sim \bar{Q} T$ and $S \sim T \bar{T}$. Those mesons are singlet under SU(3) and transforms as $\mathbf{1}+\mathbf{2 4}$, $\mathbf{5}, \overline{\mathbf{5}}$ and $\mathbf{1}$ under $\mathrm{SU}(5)_{\mathrm{GUT}}$, respectively. Dual quarks $q, t$ and anti-quarks $\bar{q}, \bar{t}$ transforms as $q:(\mathbf{3}, \mathbf{5}), t:(\mathbf{3}, \mathbf{1}), \bar{q}:(\overline{\mathbf{3}}, \overline{\mathbf{5}})$ and $\bar{t}:(\overline{\mathbf{3}}, \mathbf{1})$ under $\mathrm{SU}(3) \times \mathrm{SU}(5)_{\mathrm{GUT}}$. The parameter $\hat{\Lambda}$ has dimension one since mesons have dimension two. Amazingly, this is almost identical to the model proposed in Ref. [1]. In the vacuum of our interest:

$$
M_{Q \bar{Q}}=\left(\begin{array}{ccccc}
0 & & & & \\
& 0 & & & \\
& & 0 & & \\
& & & v^{2} & \\
& & & & v^{2}
\end{array}\right),
$$

$\mathrm{SU}(5)_{\mathrm{GUT}}$ is broken down to the standard model gauge group, and two of dual quarks in $q$ and $\bar{q}$ obtain masses. After integrating out the massive quarks, the theory becomes the SU(3) gauge 
theory with four flavors, which is confining theory with superpotential 20]:

$$
\begin{aligned}
W & =m \operatorname{Tr} M_{Q \bar{Q}}-\frac{1}{M} \operatorname{Tr}\left(M_{Q \bar{Q}} M_{Q \bar{Q}}\right)+\cdots \\
& +\frac{1}{\hat{\Lambda}} M_{Q \bar{Q}}^{(3 \times 3)} M_{q \bar{q}}^{(3 \times 3)}+\frac{1}{\hat{\Lambda}} H_{C} \bar{H}_{C}^{\prime}+\frac{1}{\hat{\Lambda}} \bar{H}_{C} H_{C}^{\prime}+\frac{1}{\hat{\Lambda}} S S^{\prime} \\
& -\frac{1}{v^{2} \hat{\Lambda}} H_{D} \bar{H}_{D} S^{\prime}+\cdots \\
& +\frac{\operatorname{det} M^{(4 \times 4)}}{\widetilde{\Lambda}^{5}}+\frac{1}{\widetilde{\Lambda}^{5}} B_{(4)} M^{(4 \times 4)} \bar{B}_{(4)} .
\end{aligned}
$$

The superscript $(3 \times 3)$ represents the $3 \times 3$ meson matrix made of colored parts of $Q \bar{Q}$ or $q \bar{q}$. The fields $H^{\prime}, \bar{H}^{\prime}$ and $S^{\prime}$ are mesons made of dual quarks; $H^{\prime} \sim q \bar{t}, H^{\prime} \sim \bar{q} t$ and $S^{\prime} \sim t \bar{t}$. The subscripts $C$ and $D$ represent $\mathrm{SU}(3)_{C}$ colored and $\mathrm{SU}(2)_{L}$ doublet part of the corresponding meson fields, respectively. $M^{(4 \times 4)}$ is the matrix:

$$
M^{(4 \times 4)}=\left(\begin{array}{c|c}
M_{q \bar{q}}^{(3 \times 3)} & H_{C}^{\prime} \\
\hline H_{C}^{\prime T} & S^{\prime}
\end{array}\right),
$$

and the baryons are

$$
B_{(4)}=\left(\begin{array}{c}
B_{C} \\
B^{-}
\end{array}\right), \quad \bar{B}_{(4)}=\left(\begin{array}{c}
\bar{B}_{C} \\
B^{+}
\end{array}\right)
$$

with the identification of $B_{C} \sim q q t, B^{-} \sim q q q, \bar{B}_{C} \sim \bar{q} \bar{q} \bar{t}$ and $B^{+} \sim \bar{q} \bar{q}$. Finally $\widetilde{\Lambda}$ is the dynamical scale of the four-flavor $\mathrm{SU}(3)$ theory.

Note here that the doublet Higgs $H_{D}$ and $\bar{H}_{D}$ do not obtain a mass term as long as $S^{\prime}=0$ whereas the colored Higgs $H_{C}$ and $\bar{H}_{C}$ already have mass terms accompanied with the dual mesons $H_{C}^{\prime}$ and $\bar{H}_{C}^{\prime}$. Indeed $S^{\prime}=0$ is ensured by the condition of $F_{S}=0$. The missing partner for the doublets, say $H_{D}^{\prime}$ and $\bar{H}_{D}^{\prime}$, have masses since the dual quarks are massive. This is the realization of the doublet-triplet splitting with infinite number of particles. If we define the $T$-number with the charge assignment $Q: 0, \bar{Q}: 0, T:+1$ and $\bar{T}:+1$, the Higgs fields $H$ and $\bar{H}$ have the same $T$-number, +1 . In this case, infinite tower of the Higgs fields should be necessary as in Fig. 1 (left). We need an extra Higgs field with charge -1 to make colored Higgs massive, but it introduces an additional unwanted massless doublet. Repeating this procedure forever is the only possibility of realizing doublet-triplet splitting in this situation. This infinite particles are realized here by the hadron tower of the Higgs fields. The mismatching of the level, i.e., no zero mode only for doublet part of $H^{\prime}$ and $\bar{H}^{\prime}$, happened because of the mass of the constituent quarks $q_{D}$ and $\bar{q}_{D}$ without violating $T$-number. A schematic picture of the hadron spectrum is shown in Fig. 1] (right).

In fact, in this $\mathrm{SU}(3)$ model, the situation is a little bit different from the story in the Introduction. If the $T$-number violating term is absent in the superpotential, the dangerous 


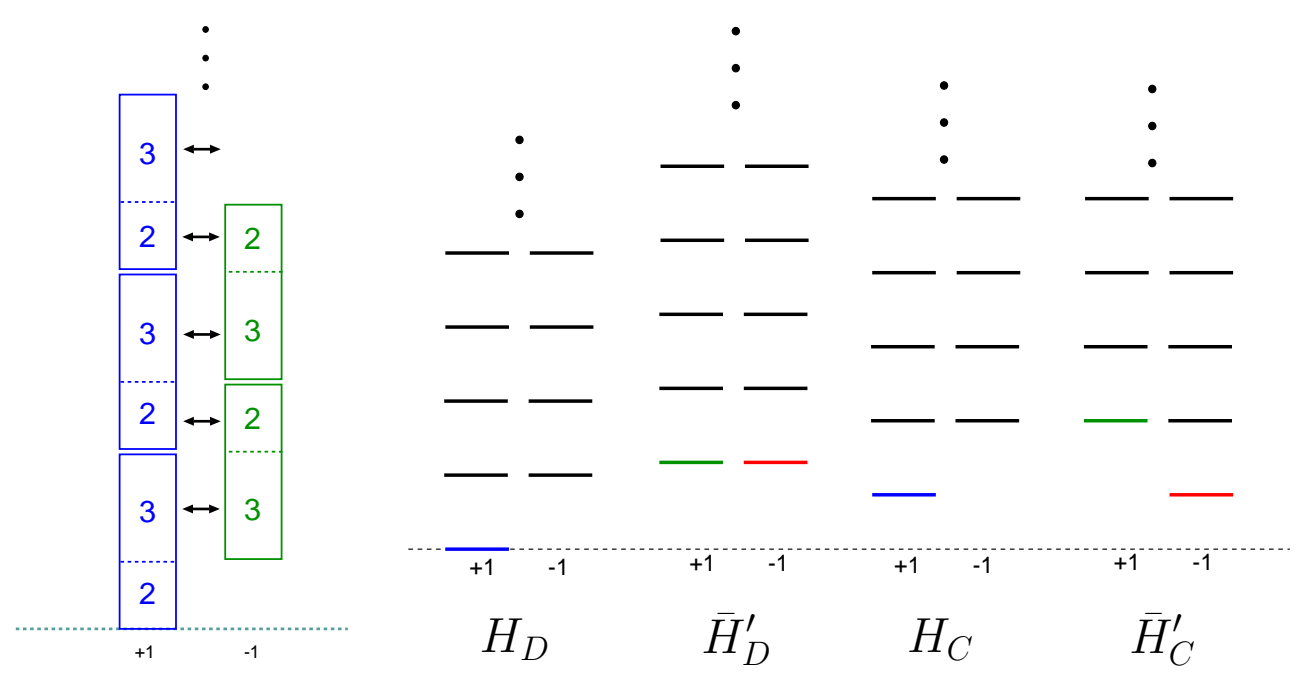

Figure 1: A schematic view of the hadron spectrum of the model. Only doublet part $H_{D}$ remains massless whereas the colored Higgs $H_{C}$ have masses by pairing up with $\bar{H}_{C}^{\prime}$

dimension-five proton-decay operators are forbidden. However, since the $T$-number is anomalous to the SU(3) gauge theory, the non-perturbatively generated superpotential in Eq. (同) violates the $T$-number. As we see later, $M_{q \bar{q}}^{(3 \times 3)}$ acquires the $\mathrm{VEV}$ and it gives $T$-number violating masses for the colored Higgs fields. Consequently, the dimension-five proton decay operators are generated by the colored-Higgs-exchange diagrams as usual.

The stability of the vacuum can be checked by solving $F=0$ conditions for all the fields. We can find a solution with Eq. (4) and

$$
\begin{aligned}
M_{q \bar{q}}^{(3 \times 3)}= & \left(\begin{array}{ll}
-m v^{2} / \hat{\Lambda} & -m v^{2} / \hat{\Lambda} \\
& -m v^{2} / \hat{\Lambda}
\end{array}\right), \\
& \frac{S}{\hat{\Lambda}}+\frac{B^{+} B^{-}}{\widetilde{\Lambda}^{5}}=-\frac{m^{3} v^{6}}{\widetilde{\Lambda}^{5} \hat{\Lambda}^{3}} .
\end{aligned}
$$

The second equation indicates that the vacuum is not uniquely determined and there is a flat direction. Correspondingly, there is a massless particle $B^{+}$and $B^{-}$which are charged under $\mathrm{U}(1)_{Y}$. This is the same situation as the classical analysis, in which the remaining gauge symmetry is just the standard model gauge group but a pair of $\mathrm{U}(1)_{Y}$ charged particles originate from $T$ and $\bar{T}$ are left massless in addition to the Higgs doublets. In order to avoid the massless charged baryons, we need to add a mass term for $T$ and $\bar{T}$, but that also makes the Higgs doublets heavy. Therefore, $N_{c}=3$ case is not acceptable.

For $N_{c}=4$, there is a hope that the exotic states confine and form standard model singlet states so that it is phenomenologically viable. However, unfortunately, it is not the case. As in 
the case of $N_{c}=3$, we can analyze the model by taking a dual gauge group and integrate out the heavy flavors. For $N_{c}=4$, the dual theory becomes $\mathrm{SU}(2)$ with four flavors. As expected, doublet-triplet splitting happens in the same way as above. By taking dual again and going back to the electric theory, we find another SU(2) theory with four flavors with superpotential interactions. The solution of the $F=0$ equations can be found with the same vacuum in Eq. (四) and (8), which gives mass terms for all the quarks except for $T$ and $\bar{T}$. The low energy theory in this vacuum is then $\mathrm{SU}(2)$ gauge theory with one flavor which has no ground state. Although we could avoid the charged exotic state, the vacuum is lifted at the quantum level.

For $N_{c} \geq 5$, the models are confining theories and acquire non-perturbatively generated superpotential. By the effect of the superpotential, there is no ground state corresponding to the vacuum with Eq. (幽).

In summary, there is no phenomenologically viable model with the particle content in Table 1 and superpotential in Eq. (1), although the doublet-triplet splitting problem is solved in a simple way. This result motivates us to consider the case with different type of groups such as $\operatorname{Sp}\left(N_{c}\right)$ and $\mathrm{SO}\left(N_{c}\right)$.

In fact, there is another interesting way of realizing massless doublet Higgs fields in this class of models. If we impose a global SU(6) symmetry in superpotential where $\mathcal{Q} \equiv(Q, T)$ and $\overline{\mathcal{Q}} \equiv(\bar{Q}, \bar{T})$ transform as $\mathbf{6}$ and $\overline{\mathbf{6}}$ and if the global symmetry is broken down to $\mathrm{SU}(4) \times \mathrm{SU}(2)$ $\times \mathrm{U}(1)$, a pair of doublet Higgs field is ensured to be massless since those are pseudo-Goldstone particles [22, 15]. With the similar superpotential:

$$
W=m \operatorname{Tr}(\mathcal{Q} \overline{\mathcal{Q}})-\frac{1}{M} \operatorname{Tr}[(\mathcal{Q} \overline{\mathcal{Q}})(\mathcal{Q} \overline{\mathcal{Q}})]+\cdots
$$

the mechanism should work and the unwanted exotic particle can be massive by the superpotential terms. We find that stable vacua exist for all $N_{c} \geq 3$. However, in this case, the dimension-five proton-decay operators are always generated even at the classical level because we cannot define conserving $T$-number. Therefore, we do not pursue this direction further.

\section{$2.2 \mathrm{Sp}\left(N_{c}\right)$ and $\mathrm{SO}\left(N_{c}\right)$ models}

We can indeed find viable models for $\operatorname{Sp}\left(N_{c}\right)$ and $\mathrm{SO}\left(N_{c}\right)$. We show the result of the analysis for these cases. The particle content of $\operatorname{Sp}\left(N_{c}\right)$ models is listed in Table 2 where the field $T_{2}$ is necessary to avoid the Witten anomaly [23]. We assumed the same superpotential for $Q$ and $\bar{Q}$ in Eq. (11) with the matrix $(Q \bar{Q})$ being the $\operatorname{Sp}\left(N_{c}\right)$ singlet combination with $5 \times 5$ flavor indices this time. Analysis can go through in the similar fashion to the $\mathrm{SU}\left(N_{c}\right)$ case, and we find that only $N_{c}=2$ case has a stable minimum with Eq. (四). The massless modes of the $\operatorname{Sp}(2)$ model 


\begin{tabular}{ccc} 
& $\mathrm{Sp}\left(N_{c}\right)$ & $\mathrm{SU}(5)_{\mathrm{GUT}}$ \\
\hline \hline$Q$ & $2 N_{c}$ & $\mathbf{5}$ \\
$\bar{Q}$ & $2 N_{c}$ & $\overline{\mathbf{5}}$ \\
$T_{1}$ & $2 N_{c}$ & $\mathbf{1}$ \\
$T_{2}$ & $2 N_{c}$ & $\mathbf{1}$ \\
\hline \hline
\end{tabular}

Table 2: The particle content of the $\operatorname{Sp}\left(N_{c}\right)$ model.

\begin{tabular}{ccc} 
& $\mathrm{SO}\left(N_{c}\right)$ & $\mathrm{SU}(5)_{\mathrm{GUT}}$ \\
\hline \hline$Q$ & $N_{c}$ & $\mathbf{5}$ \\
$\bar{Q}$ & $N_{c}$ & $\overline{\mathbf{5}}$ \\
$T$ & $N_{c}$ & $\mathbf{1}$ \\
\hline \hline
\end{tabular}

Table 3: The particle content of the $\mathrm{SO}\left(N_{c}\right)$ model.

consist of four Higgs doublets, $H_{D} \sim Q_{D} T_{1}, \bar{H}_{D} \sim \bar{Q}_{D} T_{1}, H_{D}^{\prime} \sim Q_{D} T_{2}$ and $\bar{H}_{D}^{\prime} \sim \bar{Q}_{D} T_{2}$. Two of them $H_{D}^{\prime}$ and $\bar{H}_{D}^{\prime}$ can be massive by adding a superpotential term $W \ni\left(Q T_{2}\right)\left(\bar{Q} T_{2}\right)$ without giving a mass for $H_{D}$ and $\bar{H}_{D}$. This is the model found in Ref. [14]. Similar to the $\mathrm{SU}\left(N_{c}\right)$ case, $\operatorname{Sp}\left(N_{c}\right)$ models with $N_{c} \geq 3$ do not have a vacuum with $\operatorname{rank}\left(M_{Q \bar{Q}}\right)=2$ due to the non-perturbatively generated superpotential [24].

The $\mathrm{SO}\left(N_{c}\right)$ model can also be constructed, and turns out to be the most interesting case. Detailed analysis will be presented in the next section. The particle content is given in Table 3 where we have to introduce only one $T$ field in contrast to the case of $\operatorname{SU}\left(N_{c}\right)$ or $\operatorname{Sp}\left(N_{c}\right)$. Again, the form of the superpotential is the same as that in Eq. (11). With the same analysis, we find that there are stable vacua for $4 \leq N_{c} \leq 9$ with massless Higgs doublet fields and there are no unwanted massless fields in low energy. The theory is asymptotically free for $N_{c} \geq 6$. Additional singlet fields under the standard model gauge group appear for $N_{c} \geq 5$.

\section{$3 \quad \mathrm{SO}(9)$ model}

We discuss in more detail the most interesting model among those in the previous section: $\mathrm{SO}(9) \times \mathrm{SU}(5)_{\text {GUT }}$ model. The phenomenological aspects of the model such as gauge coupling unification, proton decay and Yukawa interactions for matter fields will be addressed. Many of those features are shared with the model of Ref. [14].

The particle content and the tree-level superpotential are defined in the previous section in Table 3 and in Eq. (1). The model is $\mathrm{SO}(9)$ gauge theory with 11 flavors which is in the conformal window 25]. This fact becomes important for the discussion on the phenomenological issues. 
We take a picture of $\Lambda \gg v$ where $\Lambda$ is the scale where the $\mathrm{SO}(9)$ gauge theory becomes strong. Since the confinement does not happen until fields get decouple, the actual confinement scale coincides with the GUT scale $v$ which is set by superpotential parameters $m$ and $M$. Therefore we have an energy region of the CFT between $M_{G} \sim v$ and $\Lambda$.

The analysis of the vacuum can be done along the same line in the $\mathrm{SU}\left(N_{c}\right)$ case. We first take dual picture of the theory which is $\mathrm{SO}(6)$ gauge theory with 11 flavors with superpotential [25]:

$$
\begin{aligned}
W & =m \operatorname{Tr} M_{Q \bar{Q}}-\frac{1}{M} \operatorname{Tr}\left(M_{Q \bar{Q}} M_{Q \bar{Q}}\right)+\cdots \\
& +\frac{1}{\hat{\Lambda}} \bar{q} M_{Q \bar{Q}} q+\frac{1}{\hat{\Lambda}} \bar{q} M_{Q Q} \bar{q}+\frac{1}{\hat{\Lambda}} q M_{\bar{Q} \bar{Q}} q \\
& +\frac{1}{\hat{\Lambda}} H \bar{q} t+\frac{1}{\hat{\Lambda}} \bar{H} q t+\frac{1}{\hat{\Lambda}} S t t
\end{aligned}
$$

where $M_{Q \bar{Q}}, M_{Q Q}$ and $M_{\bar{Q} \bar{Q}}$ are mesons made of $Q$ and $\bar{Q}$ which are singlet under $\mathrm{SO}(6)$ and $\mathbf{1}+\mathbf{2 4}, \mathbf{1 5}$ and $\overline{\mathbf{1 5}}$ under $\mathrm{SU}(5)_{\mathrm{GUT}}$. The mesons involving $T$ are $H \sim Q T, \bar{H} \sim \bar{Q} T$ and $S \sim T T$, and those are $\mathbf{5}, \overline{\mathbf{5}}$ and $\mathbf{1}$ under $\mathrm{SU}(5)_{\mathrm{GUT}}$. The dual quarks $q, \bar{q}$ and $t$ transform as $q:(\mathbf{6}, \mathbf{5}), \bar{q}:(\mathbf{6}, \overline{\mathbf{5}})$ and $t:(\mathbf{6}, \mathbf{1})$ under $\mathrm{SO}(6) \times \mathrm{SU}(5)_{\text {GUT }}$. Again $\hat{\Lambda}$ is introduced such that dimensionality of the superpotential is correct. It is interesting that this dual picture is similar to the $\mathrm{SO}(6) \times \mathrm{SO}(10)_{\text {GUT }}$ model proposed in Ref. [12]. By the VEV of $M_{Q \bar{Q}}$ in Eq. (田), four flavors (doublet part of $q$ and $\bar{q}$ ) obtain masses and the low energy theory becomes the $\mathrm{SO}(6)$ theory with seven flavors (colored part of $q$ and $\bar{q}$ and $t$ ). This is still interacting theory.

When we take the dual again, the theory now comes back to the original electric theory but the gauge group is reduced to $\mathrm{SO}(5)$. The superpotential is:

$$
\begin{aligned}
W & =m \operatorname{Tr} M_{Q \bar{Q}}-\frac{1}{M} \operatorname{Tr}\left(M_{Q \bar{Q}} M_{Q \bar{Q}}\right)+\cdots \\
& +\frac{1}{\hat{\Lambda}} M_{Q \bar{Q}}^{(3 \times 3)} M_{q \bar{q}}^{(3 \times 3)}+\cdots \\
& -\frac{1}{v^{2} \hat{\Lambda}} H_{D} \bar{H}_{D} S^{\prime}+\frac{1}{\hat{\Lambda}} H_{C} \bar{H}_{C}^{\prime}+\frac{1}{\hat{\Lambda}} \bar{H}_{C} H_{C}^{\prime}+\frac{1}{\hat{\Lambda}} S S^{\prime} \\
& -\frac{1}{\hat{\Lambda}} \widetilde{Q}_{C} M_{q \bar{q}}^{(3 \times 3)} \widetilde{Q}_{C}+\cdots \\
& -\frac{1}{\hat{\Lambda}} \widetilde{Q}_{C} H_{C}^{\prime} \widetilde{T}-\frac{1}{\hat{\Lambda}} \widetilde{Q}_{C} \bar{H}_{C}^{\prime} \widetilde{T}-\frac{1}{\hat{\Lambda}} S^{\prime} \widetilde{T} \widetilde{T}
\end{aligned}
$$

where we wrote down only terms relevant for the discussion. The quarks $\widetilde{Q}, \widetilde{Q}$ and $\widetilde{T}$ will be identified with original quarks upon integrating out massive fields. The doublet-triplet splitting happens in the same way as the $\mathrm{SU}\left(N_{c}\right)$ examples. The massless doublet is obtained with $S^{\prime}=0$ which is ensured by the $F_{S}=0$ condition, and the triplets get masses by pairing with dual mesons. In contrast to the case of the SU(3) model in the previous section, there is no non-perturbatively generated superpotential. Therefore, symmetry of the superpotential at the 
classical level, the $T$-number $(T:+1)$, is respected. This is exactly the situation discussed in the Introduction. This fact becomes important in the discussion of the proton decay.

By solving the $F=0$ conditions, we can find a solution in Eqs. (4) and (8) that gives a mass term for $\widetilde{Q}_{C}$ and $\widetilde{Q}_{C}$. After integrating out the heavy fields, the theory ends up with $\operatorname{SO}(5)$ theory with one flavor $\widetilde{T}$ without superpotential and massless Higgs doublet fields. This result is the same as the classical analysis in the Higgs phase.

The decoupling of the fields immediately make the $\mathrm{SO}(5)$ interaction strong and the quark $\widetilde{T}$ confines. This $\mathrm{SO}(5)$ one flavor theory has known to have two branches [25]. In one branch, superpotential $W=\left(\widetilde{\Lambda}^{8} / \widetilde{S}\right)^{1 / 2}$ is generated with $\widetilde{\Lambda}$ being the dynamical scale of one flavor $\mathrm{SO}(5)$ theory and $\widetilde{S} \sim \widetilde{T} \widetilde{T}$. This branch is unacceptable because there is no ground state. In the other branch, no superpotential is generated and there is no singularity at the origin of meson $\widetilde{S}$ even though the gauge symmetry is enhanced there at the classical level. Therefore there is a stable vacuum in this branch. The low energy spectrum is just a pair of doublet Higgs fields with massless meson $\widetilde{S}$ and the superpotential is $W=0$.

Now we start to discuss the phenomenological issues. First, we need to check whether the gauge coupling unification is maintained in this model. The first order answer to this question is yes. There is no exotic massless fields in the spectrum, and the running of the gauge coupling constants are the same as that of the minimal supersymmetric standard model. However, the threshold correction is also important for the precise unification, that is not obvious. The question depends on the spectrum of the heavy fields and that cannot be estimated without the knowledge of the Kähler potential. However, the qualitative discussion is still possible by assuming that the Kähler potential is not very different from the classical one. In this case, the mass spectrum can be estimated by explicitly calculating the mass of the components in $Q$ and $\bar{Q}$ at the classical level. There are three classes of fields: fields eaten by the $\mathrm{SO}(9) / \mathrm{SO}(5)$ gauge fields, ones eaten by the $\mathrm{SU}(5)_{\mathrm{GUT}} /\left(\mathrm{SU}(3)_{C} \times \mathrm{SU}(2)_{L} \times \mathrm{U}(1)_{Y}\right)$ gauge fields, and others obtain masses by superpotential. The first two classes of fields have masses of order $v=\sqrt{m M / 2}$ and the last one have $O(m)$. Therefore, in order not to destroy the gauge coupling unification due to the mass splitting between those two, the mass parameter $m$ is required to be around the GUT scale $M_{G} \sim v$ which means the scale $M$ should also be of the order of the GUT scale.

This sounds unreasonable since we expect that the higher dimensional operators to be suppressed by the Plank scale $M_{\mathrm{Pl}}$ that is two order of magnitude larger than the GUT scale. Also, with GUT scale suppressed operators, we are not allowed to discuss the high energy theory above the GUT scale, which we are doing. However, in this model, the mechanism of suppressing $M$ is already built in. As we discussed before, this $\mathrm{SO}(9)$ theory is in the conformal window, and 
we expect an energy range of CFT above the GUT scale. In this case, meson fields have large negative anomalous dimension which enhances the couplings in low energy. In other words, the interaction never gets strong in high energy. The anomalous dimension of the meson fields are calculated by using the relation between the non-anomalous $R$-charge and the dimension of the operator as follows [21]:

$$
\gamma(Q \bar{Q})=D(Q \bar{Q})-2=\frac{3}{2} R(Q \bar{Q})-2=-\frac{10}{11} .
$$

With this anomalous dimension, the coefficient $1 / M$ enhances almost quadratically with scale towards low energy. Therefore it is natural to have a large enhancement. If we assume that the original operator is suppressed by the Plank scale $M_{\mathrm{Pl}}$, the factor of 100 enhancement is easily realized by a small CFT range such as from $10^{16} \mathrm{GeV}$ to $10^{17} \mathrm{GeV}$. In the same way, the coefficient $m$ enhances almost linearly with energy scale towards low energy. Therefore, the original scale of the model $m$ evaluated at the Planck scale was smaller than the GUT scale such as factor of 10 or so. This is interesting scale for the right-handed neutrino masses in the see-saw model [26].

The gauge coupling of $\mathrm{SU}(5)_{\mathrm{GUT}}$ above the GUT scale can be in the perturbative region all the way up to the Planck scale even accounting for the large anomalous dimension of $Q$ and $\bar{Q} 27$.

The Yukawa coupling constants between matters and the Higgs fields originate from higher dimensional operators since the Higgs fields are identified with the meson fields. The gauge invariant terms:

$$
W_{\text {Yukawa }}=\frac{f_{u}}{M_{Y}}(\mathbf{1 0})(\mathbf{1 0})(Q T)+\frac{f_{d}}{M_{Y}}(\mathbf{1 0})(\overline{\mathbf{5}})(\bar{Q} T),
$$

become the Yukawa interactions in low energy. The matter fields are represented by (10) and $(\overline{\mathbf{5}})$. The low energy Yukawa coupling is roughly $y_{u} \sim f_{u} M_{G} / M_{Y}$ and $y_{d} \sim f_{d} M_{G} / M_{Y}$, where $M_{G}$ is the GUT scale. The Yukawa coupling constant for the top quark is necessary to be $O(1)$, which again requires that the scale $M_{Y}$ to be the GUT scale. This is not a problem with the same reason before. The coefficient is enhanced linearly in low energy by the large anomalous dimensions.

After integrating out the massive fields with the Yukawa interactions in Eq. (14), final low energy effective superpotential is:

$$
W=W_{\mathrm{MSSM}}+\frac{y_{u} y_{d}}{m} \frac{\widetilde{S}}{M_{G}^{2}}(\hat{Q} \hat{Q} \hat{Q} \hat{L}+\hat{U} \hat{U} \hat{D} \hat{E}+\hat{Q} \hat{Q} \hat{U} \hat{D}+\hat{U} \hat{E} \hat{Q} \hat{L})
$$

with $\hat{Q}, \hat{U}, \hat{D}$ being the quark superfields and $\hat{L}, \hat{E}$ are the lepton superfields. In addition to the usual Yukawa interactions $W_{\mathrm{MSSM}}$, the baryon-number-violating terms (first two terms in 
the parenthesis) appeared. However, as long as $\widetilde{S}$ is stabilized near the origin, these terms do not cause rapid proton decays. The particle content of the low energy effective theory is just that of the minimal supersymmetric standard model and a singlet field $\widetilde{S}$ which only couples to the higher dimensional operators. The value of $\widetilde{S}$ depends on the shape of the Kähler potential and how supersymmetry is broken.

\section{$4 \quad \mu$-term driven supersymmetry breaking}

In the previous section, we have seen that the $\mathrm{SO}(9)$ model is quite successful in obtaining the massless Higgs fields in low energy spectrum. However, to be phenomenologically completely viable, the Higgs fields has to have a mass term of $O(100 \mathrm{GeV})$, the $\mu$-term, otherwise Higgsinos become massless and that is excluded by the experiments. As we see below, it is possible to obtain $\mu$-term by giving a small mass term for $T$, but it may cause a dramatic effect in the dynamical system: dynamical supersymmetry breaking [28, 29].

The $\mu$-term can be obtained by adding a superpotential term:

$$
W \ni \hat{\mu} T T
$$

which becomes $\hat{\mu} S$ in Eq. (12). With this term, the $F_{S}=0$ condition leads to

$$
S^{\prime}=-\hat{\mu} \hat{\Lambda}
$$

and it induces the mass term for the Higgs doublets:

$$
W \ni \frac{\hat{\mu}}{v^{2}} H_{D} \bar{H}_{D}
$$

With the canonically normalized fields $\hat{H}_{D}$ and $\hat{\bar{H}}_{D}$, this is nothing but the $\mu$-term, $\mu \hat{H}_{D} \hat{\bar{H}}_{D}$ with $\mu \sim \hat{\mu}$. It is obvious that the mesons made of $T$ become massive once we introduce the mass term for the quark $T$. This is also easy to understand in the classical level analysis. Since the Higgs fields are simply the components in $T$, the term in Eq. (16) is the $\mu$-term.

The term in Eq. (16) also gives a potential term for $\widetilde{S}$. With Eq. (17) and superpotential in Eq. (12), $\widetilde{T}$ obtains a term in low energy:

$$
\hat{\mu} \widetilde{T} \widetilde{T}
$$

and after confinement of the $\mathrm{SO}(5)$ gauge theory, it becomes the linear superpotential for the meson $\widetilde{S} \sim \widetilde{T} \widetilde{T}:$

$$
W \ni \hat{\mu} \widetilde{S}
$$


If we ignore the higher dimensional operators in Eq. (15), there is no solution for $F_{\tilde{S}}=0$.

Interestingly, this does not mean that the vacuum is destabilized or quarks and leptons must condense by the presence of the small $\mu$-term. First, we start the discussion with ignoring the higher dimensional operators. In this case, it is shown by Intriligator, Seiberg and Shih [18] that the vacuum is meta-stable and supersymmetry is broken there. The argument is pretty easy. Whether the vacuum is stable or not depends on the shape of the Kähler potential for $\widetilde{S}$, but since we know that for large $\widetilde{S}$, where the classical analysis is valid, the potential grows by the mass term. Therefore, there must be a local minimum somewhere. True supersymmetric vacua exist in the other branch where $\tilde{S}$ is stabilized far away from the origin $M_{G}\left(M_{G} / \hat{\mu}\right)^{2 / 3}$ (this is meaningless because it is much larger than the Planck scale) and also at different vacua from that in Eq. (4). Since the true vacua exist far away or energy difference between the true vacuum and the meta-stable one is much smaller than the height of the potential barrier of $O\left(M_{G}^{4}\right)$, we expect that the life-time of this meta-stable vacuum is long enough for us [30].

Once we include the higher dimensional operators, another supersymmetric vacuum appears where quarks and leptons acquire VEVs. However, if we assume that the scalar components of quarks and leptons obtain positive supersymmetry breaking mass-squared terms, the vacuum is again meta-stable and its life-time is very long since the peak of the potential barrier locates far from the origin $Q \sim L \sim\left(\mu M_{G}\right)^{1 / 2}$ compared to the height of the potential $V^{1 / 4} \sim$ $O\left(\mu^{3} M_{G}\right)^{1 / 4}$ [31. Therefore, we conclude that there is a supersymmetry broken meta-stable vacuum.

The size of supersymmetry breaking is $F_{\hat{S}} \sim O\left(\mu M_{G}\right)$ with canonically normalized field $\hat{S} \sim M_{G} \widetilde{S}$. For the Higgs fields, terms in the Kähler potential such as $\hat{S}^{\dagger} \hat{S} H^{\dagger} H / \tilde{\Lambda}^{2}$ may be generated by the non-perturbative effect and supersymmetry breaking can be mediated directly (in the sense of gravity mediation). In this case, the soft scalar masses for the Higgs fields are obtained with similar size to the $\mu$-term. If the term $\hat{S} H^{\dagger} H / \tilde{\Lambda}+$ h.c. is generated, which should be possible since there is no unbroken symmetry to protect the term, the trilinear $A$ and the bilinear $B$-term is also non-vanishing and the same order with the $\mu$-term. If the matters in the third generation couples to the Higgs fields strongly, the soft scalar masses for those fields can also be obtained directly.

The gauge mediation [32] also happens if $\hat{S}$ is stabilized away from the origin, where the colored-Higgs fields play a role of the messenger field.* By integrating out $\widetilde{Q}_{C} \widetilde{\widetilde{Q}}_{C}$ in Eq. (12), we obtain mass terms for the colored-Higgs fields:

$$
W \simeq \hat{S} \hat{H}_{C}^{\prime} \hat{\bar{H}}_{C}^{\prime}+M_{C} \hat{H}_{C} \hat{\bar{H}}_{C}^{\prime}+M_{C} \hat{\bar{H}}_{C} \hat{H}_{C}^{\prime},
$$

*A similar structure of the model can be found in Ref. 33. 
where $M_{C}$ is the colored-Higgs mass of order $M_{G}$ and meson fields are canonically normalized. Unfortunately, with this structure of superpotential, the leading order contribution to the gaugino masses of $O\left(F_{\hat{S}} /\langle\hat{S}\rangle\right)$ cancels out [34] $]^{\dagger}$, and moreover there is no contribution to the $\mathrm{SU}(2)_{L}$ gauginos.

In order to obtain the gaugino masses, there must be the gauge-mediation effect since the supersymmetry breaking scale is too low $\left(O\left(\mu M_{G}\right)\right)$ for the gravity mediation. A simple example for the gauge mediation is to assume an interaction term:

$$
W_{\text {messenger }}=\frac{1}{M_{X}} T^{2} \Phi \bar{\Phi}
$$

where $\Phi$ and $\bar{\Phi}$ transform under the $\mathrm{SU}(5)_{\text {GUT }}$ such as $\mathbf{5}$ and $\overline{\mathbf{5}}$ and singlet under $\mathrm{SO}(9)$. By the enhancement of the $1 / M_{X}$ suppressed term due to large anomalous dimension of $T^{2}$, this term effectively becomes

$$
W_{\text {messenger }} \rightarrow \lambda \hat{S} \Phi \bar{\Phi}
$$

with $\lambda \sim O(0.1-1)$ even if $M_{X}$ is $O\left(M_{\mathrm{Pl}}\right)$. With this superpotential, another supersymmetric true vacuum with $\Phi=\bar{\Phi} \neq 0$ appears. However, assuming that the $\hat{S}$ field is stabilized away from the origin, which is reasonable since we expect the presence of the linear term in the Kähler potential, the vacuum with $\Phi=\bar{\Phi}=0$ is meta-stable 35. At the meta-stable vacuum the gaugino masses are generated to be

$$
m_{1 / 2}=\frac{\alpha}{4 \pi} \frac{F_{\hat{S}}}{\langle\hat{S}\rangle}
$$

In order for the gaugino masses to be similar to $\mu$ in size, somewhat small value of $\langle\hat{S}\rangle \sim$ $10^{14-15} \mathrm{GeV}$ is necessary. That is consistent with the suppression of the coefficient of protondecay operators in Eq. (15).

It might be possible and would be great if all the gaugino masses are obtained by the (really) direct gauge mediation [36] without having the messenger particles above by extending the gauge group and/or matter content. But in any case, the pattern of the supersymmetry breaking parameters in this scenario is essentially the gauge-mediation type except for the Higgs sector, since the Higgs fields can feel the supersymmetry breaking directly. The soft scalar masses $m_{H_{u}}^{2}$, $m_{H_{d}}^{2}, \mu, B$ and $A$-terms can be taken as free parameters at the GUT scale. (Probably $m_{H_{u}}^{2} \simeq$ $m_{H_{d}}^{2}$ because of the parity symmetry $Q \leftrightarrow \bar{Q}$ which is only broken by the Yukawa interactions.) The soft masses for the third generation fields may also be modified. This prediction should be able to be tested at future colliders. The source of flavor and CP violation in this model

\footnotetext{
${ }^{\dagger}$ I thank Y. Nomura for discussion on this point.
} 
is only in the Yukawa coupling constants, which is desired situation taking into account the stringent constraints on the supersymmetry breaking parameters. The gravity-mediation effect gives $O(1 \%)$ correction to the parameters. This might be interesting for the detection of the flavor and $\mathrm{CP}$ violating processes ${ }^{\ddagger}$.

The lightest supersymmetric particle is the gravitino. The mass is estimated to be

$$
m_{3 / 2}=\frac{F_{\hat{S}}}{\sqrt{3} M_{\mathrm{Pl}}} \sim \mu\left(\frac{M_{G}}{M_{\mathrm{Pl}}}\right) \sim O(1) \mathrm{GeV} .
$$

This mass range is interesting for cosmology [38] and also for collider experiments [39].

\section{Gravitational supersymmetry breaking}

Because of the large anomalous dimension, the size of $\mu$-parameter is originally smaller than $O(100 \mathrm{GeV})$ by a factor of 10 or so. This is about the size of the gravitino mass. Therefore it is possible that the origin of the $\mu$-term can actually be the cosmological constant by the Giudice-Masiero mechanism [17]. Assuming a presence of the $T^{2}$ term in the Kähler potential, this effectively becomes the $\hat{\mu}$-term in the superpotential. It is equivalent to study a model with

$$
K=\hat{S}^{\dagger} \hat{S}-a \widetilde{\Lambda}(\hat{S}+\text { h.c. })-\frac{\left(\hat{S}^{\dagger} \hat{S}\right)^{2}}{\widetilde{\Lambda}^{2}}+\cdots \quad \text { and } \quad W=c
$$

where $c$ is a constant term which represents the negative cosmological constant of order $c^{2} \sim m_{3 / 2}^{2}$ in the unit of $M_{\mathrm{Pl}}=1$, which is always necessary to cancel the positive vacuum energy from the supersymmetry breaking. The parameters have a hierarchical structure: $a \gg 1, \widetilde{\Lambda} \ll 1$ and $c \ll 1$, where $a$ represents the enhancement of the coupling through the large anomalous dimension. By the Kähler transformation, this is identical to the system:

$$
K=\hat{S}^{\dagger} \hat{S}-\frac{\left(\hat{S}^{\dagger} \hat{S}\right)^{2}}{\widetilde{\Lambda}^{2}}+\cdots \quad \text { and } \quad W=c e^{a \widetilde{\Lambda} \hat{S}} .
$$

By expanding the superpotential, we obtain the $\hat{\mu}$-term of order $a c \simeq a m_{3 / 2}$. Within the range $|\hat{S}| \lesssim \widetilde{\Lambda}$, where the effective theory makes sence, the minimum of the potential exists near the origin $\hat{S} \simeq \widetilde{\Lambda} /(4 a)$, and the cosmological constant can be cancelled when $a \simeq \sqrt{3} / \widetilde{\Lambda} \sim O(100)$. Supersymmetry is broken at the minimum with $F_{\hat{S}} \simeq a c \widetilde{\Lambda} \simeq \mu \widetilde{\Lambda}$. The value of $\hat{S}$ at the minimum is $O\left(10^{14} \mathrm{GeV}\right)$ which is consistent with the required value for the gauge mediation in Eq. (24) and also the suppression of the coefficient of the dimension-five proton-decay operators in Eq. (15).

\footnotetext{
${ }^{\ddagger}$ There might be a suppression of the gravity-mediation effect by the conformal sequestering [37].
} 


\section{Discussion and Conclusions}

From the consideration of the mystery of the Higgs particle, we arrived at a rather unified picture. At every stage of the phase transitions, GUT breaking, supersymmetry breaking and electroweak symmetry breaking, the Higgs field may be playing a crucial role. We have succeeded to construct a realistic GUT models with dynamical symmetry breaking, and found that, in the $\mathrm{SO}(9)$ model, the inclusion of the $\mu$-term for the Higgs fields triggers to break supersymmetry in meta-stable vacua by the same dynamics.

We discuss possible generalizations of the model. Although we discussed the GUT breaking and supersymmetry breaking in a unified picture, we can separately discuss the following mechanisms:

- Doublet-triplet splitting through dynamical GUT breaking,

- $\mu$-term driven supersymmetry breaking.

The dynamical GUT breaking without supersymmetry breaking is possible. The $\mu$-term can be obtained from separate SUSY breaking sector by, e.g., the Giudice-Masiero mechanism. The $\mathrm{SO}(10)_{\text {GUT }}$ extension of the model should be straightforward and is interesting for the discussion of the neutrino masses. Considering different types of particle content and assumption on the superpotential is also worth investigating.

It is possible to obtain massless colored Higgs fields instead of the doublet fields. In this case, the doublet-triplet splitting can be done by introducing a pair of elementary Higgs fields with the coupling to the meson operators [10, 11, 12, 13]. This is possible in the $\operatorname{rank}\left(M_{Q \bar{Q}}\right)=3$ vacuum with $\mathrm{SO}\left(N_{c}\right)$ with $6 \leq N_{c} \leq 11$.

The $\mu$-term driven supersymmetry breaking can be discussed without GUT. For example, in the $\mathrm{SO}(9)$ model we can gauge only $\mathrm{SU}(2)_{L} \times \mathrm{U}(1)_{Y}$ subgroup of $\mathrm{SU}(5)_{\mathrm{GUT}}$. Then the dynamical scale can be lowered (or even raised) as long as the gauge couplings of the standard model gauge group maintains the perturbativity. Adding $\mu$-term breaks supersymmetry in the same way but the scale can be much lower (or higher). The gauge mediation through the colored-Higgs fields might be able to be generalized as a realistic direct gauge-mediation model. We leave those questions for future studies. In any case, the prediction to the low energy spectrum is a modification of the gauge-mediation type in the Higgs sector. In the $\mathrm{SO}(9)$ model we presented,

there is a cosmological problem associated with the modulus $\hat{S}$ 40. The mass of $\hat{S}$ is of the same order of $\mu$, i.e., $O(100 \mathrm{GeV})$ independent of the dynamical scale. A realistic cosmological scenario needs to be considered. 
We see that two mechanisms non-trivially fit into a picture: dynamical GUT and supersymmetry breaking. Although it is not likely that we can directly probe the GUT theory by experiments, the spectrum of the supersymmetric particles in low energy may give us a hint for the high energy theories. In this model, the direct connection between the Higgs fields and the supersymmetry breaking sector may provide a characteristic feature in the low energy spectrum.

\section{Acknowledgments}

The work of R.K. was supported by the U.S. Department of Energy under contract number DE-AC02-76SF00515.

\section{References}

[1] S. Dimopoulos and H. Georgi, Nucl. Phys. B 193, 150 (1981); S. Dimopoulos, S. Raby and F. Wilczek, Phys. Rev. D 24, 1681 (1981). N. Sakai, Z. Phys. C 11, 153 (1981).

[2] H. Georgi and S. L. Glashow, Phys. Rev. Lett. 32, 438 (1974).

[3] S. Weinberg, Phys. Rev. D 26, 287 (1982); N. Sakai and T. Yanagida, Nucl. Phys. B 197, $533(1982)$.

[4] T. Goto and T. Nihei, Phys. Rev. D 59, 115009 (1999) [arXiv:hep-ph/9808255]; H. Murayama and A. Pierce, Phys. Rev. D 65, 055009 (2002) [arXiv:hep-ph/0108104].

[5] T. Watari, talk at the KEK theory meeting on Collider Physics, Tsukuba, Japan, February 25-27, 2002.

[6] Y. Kawamura, Prog. Theor. Phys. 105, 999 (2001) [arXiv:hep-ph/0012125].

[7] G. Altarelli and F. Feruglio, Phys. Lett. B 511, 257 (2001) [arXiv:hep-ph/0102301]; L. J. Hall and Y. Nomura, Phys. Rev. D 64, 055003 (2001) [arXiv:hep-ph/0103125].

[8] J. M. Maldacena, Adv. Theor. Math. Phys. 2, 231 (1998) [Int. J. Theor. Phys. 38, 1113 (1999)] [arXiv:hep-th/9711200]; S. S. Gubser, I. R. Klebanov and A. M. Polyakov, Phys. Lett. B 428, 105 (1998) [arXiv:hep-th/9802109]; E. Witten, Adv. Theor. Math. Phys. 2, 253 (1998) [arXiv:hep-th/9802150].

[9] N. Arkani-Hamed, M. Porrati and L. Randall, JHEP 0108, 017 (2001) [arXiv:hepth/0012148]; R. Rattazzi and A. Zaffaroni, JHEP 0104, 021 (2001) [arXiv:hep-th/0012248]. 
[10] T. Yanagida, Phys. Lett. B 344, 211 (1995) [arXiv:hep-ph/9409329]; T. Hotta, K. I. Izawa and T. Yanagida, Phys. Rev. D 53, 3913 (1996) [arXiv:hep-ph/9509201]; J. Hisano and T. Yanagida, Mod. Phys. Lett. A 10, 3097 (1995) [arXiv:hep-ph/9510277]; K. I. Izawa and T. Yanagida, Prog. Theor. Phys. 97, 913 (1997) [arXiv:hep-ph/9703350].

[11] T. Hotta, K. I. Izawa and T. Yanagida, Phys. Lett. B 409, 245 (1997) [arXiv:hepph/9511431]; T. Hotta, K. I. Izawa and T. Yanagida, Prog. Theor. Phys. 95, 949 (1996) [arXiv:hep-ph/9601320].

[12] T. Hotta, K. I. Izawa and T. Yanagida, Phys. Rev. D 54, 6970 (1996) [arXiv:hep$\mathrm{ph} / 9602439]$.

[13] K. I. Izawa and T. Yanagida, Prog. Theor. Phys. 99, 423 (1998) [arXiv:hep-ph/9710218].

[14] R. Kitano and G. D. Kribs, JHEP 0503, 033 (2005) [arXiv:hep-ph/0501047].

[15] Y. Nomura, D. Poland and B. Tweedie, arXiv:hep-ph/0605014.

[16] A. H. Chamseddine, R. Arnowitt and P. Nath, Phys. Rev. Lett. 49, 970 (1982); R. Barbieri, S. Ferrara and C. A. Savoy, Phys. Lett. B 119, 343 (1982); L. J. Hall, J. D. Lykken and S. Weinberg, Phys. Rev. D 27 (1983) 2359.

[17] G. F. Giudice and A. Masiero, Phys. Lett. B 206, 480 (1988); J. A. Casas and C. Munoz, Phys. Lett. B 306, 288 (1993) [arXiv:hep-ph/9302227].

[18] K. Intriligator, N. Seiberg and D. Shih, JHEP 0604, 021 (2006) [arXiv:hep-th/0602239].

[19] R. Barbieri, G. R. Dvali and A. Strumia, Phys. Lett. B 333, 79 (1994) [arXiv:hep$\mathrm{ph} / 9404278]$.

[20] N. Seiberg, Phys. Rev. D 49, 6857 (1994) [arXiv:hep-th/9402044]; K. A. Intriligator, R. G. Leigh and N. Seiberg, Phys. Rev. D 50, 1092 (1994) [arXiv:hep-th/9403198].

[21] N. Seiberg, Nucl. Phys. B 435, 129 (1995) [arXiv:hep-th/9411149].

[22] K. Inoue, A. Kakuto and H. Takano, Prog. Theor. Phys. 75, 664 (1986).

[23] E. Witten, Phys. Lett. B 117, 324 (1982).

[24] K. A. Intriligator and P. Pouliot, Phys. Lett. B 353, 471 (1995) [arXiv:hep-th/9505006].

[25] K. A. Intriligator and N. Seiberg, Nucl. Phys. B 444, 125 (1995) [arXiv:hep-th/9503179]. 
[26] T. Yanagida, in Workshop on the Unified Theory and the Baryon Number in the Universe, Tsukuba, Japan, Feb 13-14, 1979, edited by O. Sawada and A. Sugamoto; M. Gell-Mann, P. Ramond and R. Slansky, Print-80-0576 (CERN).

[27] V. A. Novikov, M. A. Shifman, A. I. Vainshtein and V. I. Zakharov, Nucl. Phys. B 229, 381 (1983); M. A. Shifman and A. I. Vainshtein, Nucl. Phys. B 277, 456 (1986) [Sov. Phys. JETP 64, 428 (1986 ZETFA,91,723-744.1986)].

[28] E. Witten, Nucl. Phys. B 188, 513 (1981).

[29] I. Affleck, M. Dine and N. Seiberg, Phys. Rev. Lett. 51, 1026 (1983); I. Affleck, M. Dine and N. Seiberg, Nucl. Phys. B 241, 493 (1984); I. Affleck, M. Dine and N. Seiberg, Nucl. Phys. B 256, 557 (1985).

[30] S. R. Coleman, Phys. Rev. D 15, 2929 (1977) [Erratum-ibid. D 16, 1248 (1977)]; C. G. . Callan and S. R. Coleman, Phys. Rev. D 16, 1762 (1977).

[31] M. J. Duncan and L. G. Jensen, Phys. Lett. B 291, 109 (1992).

[32] M. Dine, A. E. Nelson and Y. Shirman, Phys. Rev. D 51, 1362 (1995) [arXiv:hepph/9408384]; M. Dine, A. E. Nelson, Y. Nir and Y. Shirman, Phys. Rev. D 53, 2658 (1996) [arXiv:hep-ph/9507378].

[33] K. I. Izawa and T. Yanagida, Prog. Theor. Phys. 114, 433 (2005) [arXiv:hep-ph/0501254].

[34] K. I. Izawa, Y. Nomura, K. Tobe and T. Yanagida, Phys. Rev. D 56, 2886 (1997) [arXiv:hep$\mathrm{ph} / 9705228]$.

[35] T. Hotta, K. I. Izawa and T. Yanagida, Phys. Rev. D 55, 415 (1997) [arXiv:hep-ph/9606203].

[36] E. Poppitz and S. P. Trivedi, Phys. Rev. D 55, 5508 (1997) [arXiv:hep-ph/9609529]; L. Randall, Nucl. Phys. B 495, 37 (1997) [arXiv:hep-ph/9612426]; N. Arkani-Hamed, J. March-Russell and H. Murayama, Nucl. Phys. B 509, 3 (1998) [arXiv:hep-ph/9701286].

[37] M. A. Luty and R. Sundrum, Phys. Rev. D 65, 066004 (2002) [arXiv:hep-th/0105137].

[38] J. L. Feng, A. Rajaraman and F. Takayama, Phys. Rev. Lett. 91, 011302 (2003) [arXiv:hepph/0302215]; J. L. Feng, S. f. Su and F. Takayama, Phys. Rev. D 70, 063514 (2004) [arXiv:hep-ph/0404198]; J. L. Feng, S. Su and F. Takayama, Phys. Rev. D 70, 075019 (2004) [arXiv:hep-ph/0404231]. 
[39] W. Buchmuller, K. Hamaguchi, M. Ratz and T. Yanagida, Phys. Lett. B 588, 90 (2004) [arXiv:hep-ph/0402179]; K. Hamaguchi, Y. Kuno, T. Nakaya and M. M. Nojiri, Phys. Rev. D 70, 115007 (2004) [arXiv:hep-ph/0409248]; J. L. Feng and B. T. Smith, Phys. Rev. D 71, 015004 (2005) [Erratum-ibid. D 71, 0109904 (2005)] [arXiv:hep-ph/0409278].

[40] T. Banks, D. B. Kaplan and A. E. Nelson, Phys. Rev. D 49, 779 (1994) [arXiv:hep$\mathrm{ph} / 9308292]$. 